\title{
Lessons learnt from the medical and psychosocial evaluation of childhood acute lymphoblastic leukemia (ALL) survivors enrolled in EORTC Children Leukemia Group Trials between 1971 and 1998 and future perspectives for long-term outcome research
} \author{
Yves Benoit ${ }^{\mathrm{e}}$ \\ ${ }^{a}$ University dept. of Pediatrics, Pediatric Onco-Hematology, CHR Citadelle, Liège, Belgium \\ ${ }^{\mathrm{b}}$ EORTC Headquarters, Brussels, Belgium \\ ${ }^{c}$ Dept. of Pediatric Onco-Hematology, Lyon University Hospital, France \\ ${ }^{\mathrm{d}}$ Dept. of Pediatric Onco-Hematology, Nice University Hospital, France \\ e Dept. of Pediatric Hematology-Oncology, Ghent University Hospital, Belgium
}

Caroline Piette $^{\mathrm{a}, *}$, Teresa de Rojas ${ }^{\mathrm{b}}$, Stefan Suciu ${ }^{\mathrm{b}}$, Michal Kicinski ${ }^{\mathrm{b}}$, Yves Bertrand ${ }^{\mathrm{c}}$, Gaetan de Schaetzen $^{\mathrm{b}}$, Caroline Gilotay ${ }^{\mathrm{b}}$, Bart Meulemans ${ }^{\mathrm{b}}$, Séraphine Rossi ${ }^{\mathrm{b}}$, Pierre Röhrlich ${ }^{\mathrm{d}}$,

\section{A R T I C L E I N F O}

\section{Keywords:}

Survivorship

Patient reported outcomes

Childhood leukemia

Socioeconomic outcome

Fertility

\begin{abstract}
A B S T R A C T
Acute lymphoblastic leukemia (ALL) is the most common childhood cancer. With dramatic improvements in survival observed since the 70's, it progressively became evident that the long-term survivors faced late morbidity, late mortality and psychosocial troubles.

In 2010, the EORTC developed the retrospective 58LAE study in order to evaluate the long-term outcome of the 2621 eligible childhood ALL survivors enrolled between 1971 and 1998.

The first sub-project project showed that ETV6-RUNX1 positive patients had better long-term outcome and had specific sensitivities to treatments. The second sub-project showed that omission of cranial radiotherapy did not increase the risk of relapse and was associated with a higher incidence of second neoplasms and late toxicities in medium and high-risk patients, without central nervous system (CNS) involvement. The third subproject identified hematopoietic stem cell transplantation, cranial radiotherapy and having a relapse as risk factors for worse socio-economic outcome. Finally, the fertility status of the survivors was also evaluated.

The 58LAE project has raised several challenges when translated into the "real-life" setting, which include the difficulties of following childhood cancer survivors throughout their transition to adult life; the statistical analysis of a cohort of patients treated in multiple clinical trials and along different years; the need for combining different approaches to gather sufficient quality patient data; and the challenge of overcoming the healthcare administrative and regulatory obstacles.

New ways of addressing survivorship studies are needed to address these challenges.
\end{abstract}

\section{The scientific background}

Acute lymphoblastic leukemia (ALL) is the most common childhood cancer. The introduction of intensive combinations of chemotherapy, including the use of stem cell transplantation, the refinement of riskbased stratification and the improvement of supportive care resulted in dramatic increase in survival since the 70's. Five-year overall survival progressively raised from $20 \%$ in $1970-85 \%$ in children ( $<14$ years) and $62 \%$ in adolescents (15-19 years) with contemporary therapy. In parallel, it progressively became evident that the long-term survivors faced late morbidity, late mortality and psychosocial troubles due to ALL and its treatments leading to a shift paradigm from survival to life of quality.

Beside large cohorts of childhood cancer survivors treated with various therapeutic protocols, the European Organization for Research and Treatment of Cancer Children's Leukemia Group (EORTC CLG) initiated a specific long-term outcome evaluation of childhood ALL survivors homogeneously treated in EORTC studies. A long-term update

\footnotetext{
* Corresponding author.

E-mail addresses: caroline.piette@eortc.be, caroline.piette@chrcitadelle.be (C. Piette).
} 
of specific randomized questions, taking into account both the longterm survival and the late adverse effects was indeed needed in order to confirm some controversial therapeutic choices, such as the indications of cranial irradiation in childhood ALL. Long-term updates were also warranted in case late relapses were suspected, such as in the ETV6RUNX1 positive or hyperdiploid ALL subgroups. Furthermore, late follow-up results are useful to orientate specific therapeutic choices and further adapt the risk-directed therapy in future protocols. Finally, they were necessary to set up specific and standardized long-term follow-up for childhood ALL patients treated in BFM-like studies.

In 2010, the EORTC CLG developed a "Late Adverse Effects" project called "EORTC 58LAE project" ("Assessment of the long-term outcome of childhood ALL patients enrolled in EORTC Children's Leukemia Group trials between 1971 and 1998”).

The aims of this retrospective project were to assess the long-term outcome of childhood ALL patients enrolled in EORTC CLG trials between 1971 and 1998:

- Long-term vital status: evaluation of the long-term survival and of the incidence of death due to ALL progression/relapse, acute toxicity, late adverse effects or second malignant neoplasms (SMN).

- Medical data: assessment of the long-term disease status, the incidence and the types of late adverse effects and SMN, and comparison between randomized treatment arms or treatment groups.

- Socioeconomic data: evaluation of the marital status, the education level and work and comparison between randomized treatment arms or treatment groups and with the general population.

- Quality of Life (QOL) data: study of the QOL level of patients of 18 years or older at the time of the 58LAE study enrollment and comparison with the general population, assessment of the impacts of the treatment type, the occurrence of late adverse effects, the occurrence of SMN and/or the socioeconomic status on QOL and health-related QOL (HRQOL).

\section{The project}

Patients with ALL or lymphoblastic lymphoma who were enrolled as children (less than 18 years old at diagnosis) in EORTC CLG studies between 1971 and 1998 (EORTC studies 58741, 58831/2 and 58881) were eligible in the 58LAE project. For the socioeconomic and QOL evaluations, eligible patients were those eligible in the EORTC study 58LAE and 18 years or older at the time of the evaluation.

Three studies have been conducted between 1971 and 1998 by the EORTC CLG, in order to evaluate the type of consolidation therapy and the type of maintenance therapy (EORTC study 58741), the use of less aggressive regimen for low risk patients (EORTC study 58831), the efficiency of high dose methotrexate to replace cranial radiotherapy for sub-clinical central nervous system (CNS) involvement for medium or high risk patients (EORTC study 58832) and the feasibility of a rapidly rotating and intensive chemotherapy regimen for very high risk patients [1-4] (EORTC study 58881) (see Table 1).

In order to avoid the sending of documents to the family of a deceased patient, the vital status of all patients was first updated (see below, level 1). For identified survivors, an "Information sheet and informed consent form", a "Questionnaire on long-term outcome after leukemia", and QOL questionnaires were sent to the patients by the institutions. Survivors were defined as:

- Alive and agreeing to take part in case they sent back a completed and signed informed consent;

- Alive and disagreeing to take part, by mail or by phone call;

- Lost to follow up in case their contact detail could not be retrieved, or in case the institutions received no answer after two attempts.

For the information that had to be updated (e.g. disease status, late adverse events, SMN or psychosocial data), data retrieval was divided

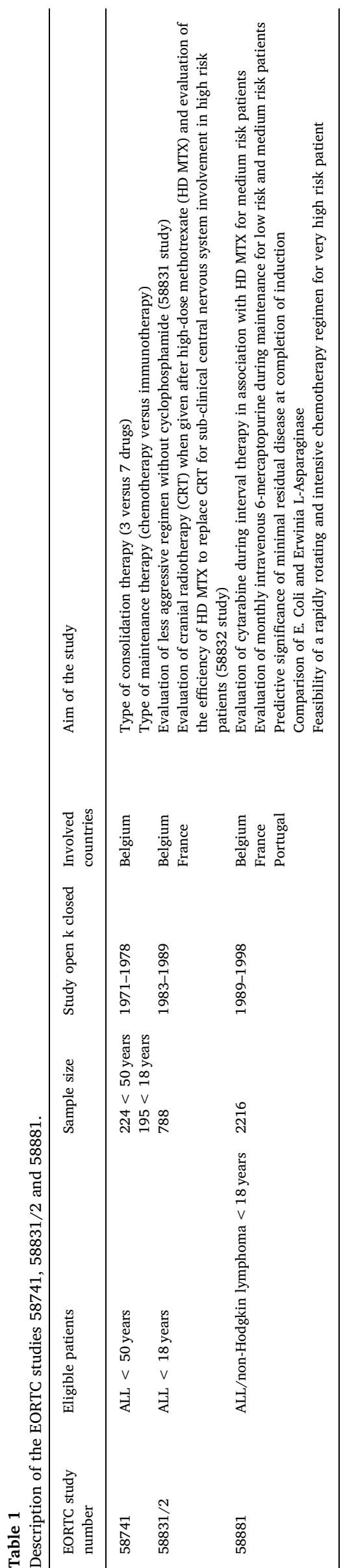


in four levels:

- Level 1 - Update of the vital status (alive, dead or lost to follow up, date last known to be alive or date of death)

The update of the vital status was done directly by the institutions based on either their own medical records or on information retrieved from the national registries at the institution's request.

\section{- Level 2 - Collection of medical data}

Medical data (disease status, late adverse effects and SMN) were retrieved by the institutions from the medical records of the patients and completed in the EORTC database by means of an electronic "Outcome form", specifically designed for the 58LAE project. The Outcome form was pre-filled with the most recent data already available in the EORTC database, in order to reduce the workload and the risk of mistakes for the institutions. New medical visit or complementary examinations were recommended when feasible, but not mandatory.

Late adverse effects were defined as adverse effects occurring 2 months or later after ending first line therapy or during the first line therapy period and persisting 2 months or more after ending first line therapy.

SMN was defined as any malignancy that occurred after the diagnosis of ALL and distinct from it, whatever the remission status of the patient. Benign tumors were not considered as SMN, except for CNS tumors.

- Level 3 - Collection of socioeconomic data from patients of 18 years or older at the time of the study

These data (marital status, education, work, insurance, puberty, fertility and offspring) were collected from identified survivors by means of a "Questionnaire on long-term outcome after leukemia", derived from the "Life Situation Questionnaire" developed by the EORTC Lymphoma group [5].

- Level 4 - Collection of QOL data from patients of 18 years or older at the time of the study

Three QOL questionnaires were used for this part of the project: the "Short Form Health Survey" (SF-12) [6], the "Impact of CancerChildhood Survivors" (IOC-CS) [7] and a qualitative instrument derived from the "Quality of Life Systemic Inventory" (QLSI) [8].

\section{The results}

A total of 32 institutions in Belgium and France took part to levels 1 and 2 (vital status and medical data), and 22 institutions also participated in levels 3 and 4 (socioeconomic and QOL data).

A total of 3228 patients were included for ALL or lymphoblastic lymphoma in EORTC CLG studies between 1971 and 1998, and 2621 were eligible in EORTC study 58LAE (update of vital status) (Fig. 1). A total of 723 patients were alive, 507 were dead and 1391 were lost to follow-up.

Medical, socioeconomic and QOL data were updated for respectively 1676,507 , and 193 patients, representing $79 \%, 36 \%$ and $14 \%$ of eligible patients (Table 2).

Different sub-projects have been completed in the framework of the EORTC study 58LAE.

The first project evaluated the long-term prognostic and predictive value of ETV6-RUNX1 in B-cell precursor (BCP)-ALL patients treated according to EORTC studies 58881 and 58951 [9]. In childhood BCPALL, the presence of an ETV6-RUNX1 fusion transcript defines one of the most prevalent genetic subgroups, and has been associated with a relatively high number of late relapses. Furthermore, although this alteration is associated with favourable outcome, several questions related to the therapeutic approach remained open. A long-term update of the EORTC CLG results was therefore warranted. In our series, the presence of ETV6-RUNX1 was associated with better outcome and with virtually no relapse after 6 years from diagnosis. ETV6-RUNX1 positive patients had specific sensitivities to treatments. They could benefit from dexamethasone during induction whereas maintenance therapy should not be intensified. These results stressed the importance of analysing homogeneous oncogenic subgroups when comparing different therapeutic schemes.

In the second sub-project, we investigated the long-term outcome, the occurrence of SMN and the incidence of late toxicities in medium and high risk patients, without CNS involvement, and randomized for receiving or not cranial radiotherapy (CRT) in the EORTC study 58832 [10]. Historically, pre-emptive cranio-spinal or CRT was a standard component of childhood ALL treatment protocols, to prevent CNS relapses. Following the emergence of CRT-related sequellae (neurocognitive, endocrine or SMN), intrathecal (IT) and systemic chemotherapy successfully replaced CRT in most protocols for standard risk ALL. However, in medium and high risk ALL patients its omission is still debatable. A total of 176 randomized patients were included in the analyses: 84 in the CRT group and 92 in the No CRT group. Omission of CRT did not increase the risk of CNS or non-CNS relapse. On long-term evaluation, CRT was associated with a higher incidence of SMN, late CNS and endocrine toxicities. These long-term results indicate that prophylactic CRT can be safely omitted in childhood medium and high risk ALL patients receiving IT and systemic chemotherapy as CNS prophylaxis.

The third sub-project assessed the long-term socioeconomic outcomes of adult survivors of childhood ALL enrolled in the three EORTC studies 58741, 58831/2 and 58881 and the effect of patient's and disease characteristics and treatment modalities [11]. Exposure to treatments may have severe toxicities such as cognitive deterioration after CRT and impaired general physical functioning after hematopoietic stem cell transplantation (HSCT). These late effects may negatively impact the socioeconomic functioning of survivors. The median time between the diagnosis and the socioeconomic evaluation was 20 years (range: 13-42). The median age at follow-up was 25 years (range: 18-53). Among survivors at school at the time of the diagnosis, $22 \%$ retook one and $6 \%$ two or more years due to the disease and $4 \%$ were unable to finish their education. Interestingly, $21 \%$ of the survivors had more and only $10 \%$ had less educational ambitions due to the disease. More than a half of the survivors had at least a bachelor (undergraduate) degree, $64 \%$ had a job and $38 \%$ were married or lived with their partner. Among those 25 years of age or older, the percentages for obtaining a bachelor degree, having a job and having a partner were $54 \%, 84 \%$ and $57 \%$, respectively. In a multivariate model, HSCT and CRT were associated with a higher probability of not obtaining a bachelor degree and HSCT was associated with not having a job. Having a relapse was associated with a higher probability of not having a partner and working half-time. This study added to the body of evidence supporting the omission of CRT when other treatment alternatives are sufficient, like in first line treatment. It also indicated the need to provide special support to the most vulnerable groups of childhood ALL survivors, in particular those who received HSCT.

Finally, we evaluated the fertility status of adult survivors of childhood ALL enrolled in the three EORTC studies 58741, 58831/2 and 58881 [12]. The impact on fertility caused by widely employed ALL treatments such as alkylating agents, cranial radiotherapy, total body irradiation and orchiectomy has indeed been an emerging concern over the last years. The median time between the diagnosis and the evaluation was 20 years (range: 13-42). The median age at follow-up was 25 years (range: 18-53). Among females who had tried to become pregnant, $89 \%$ had been pregnant, $75 \%$ had children and $12 \%$ used medical help to become pregnant. One fifth of the ever-pregnant 

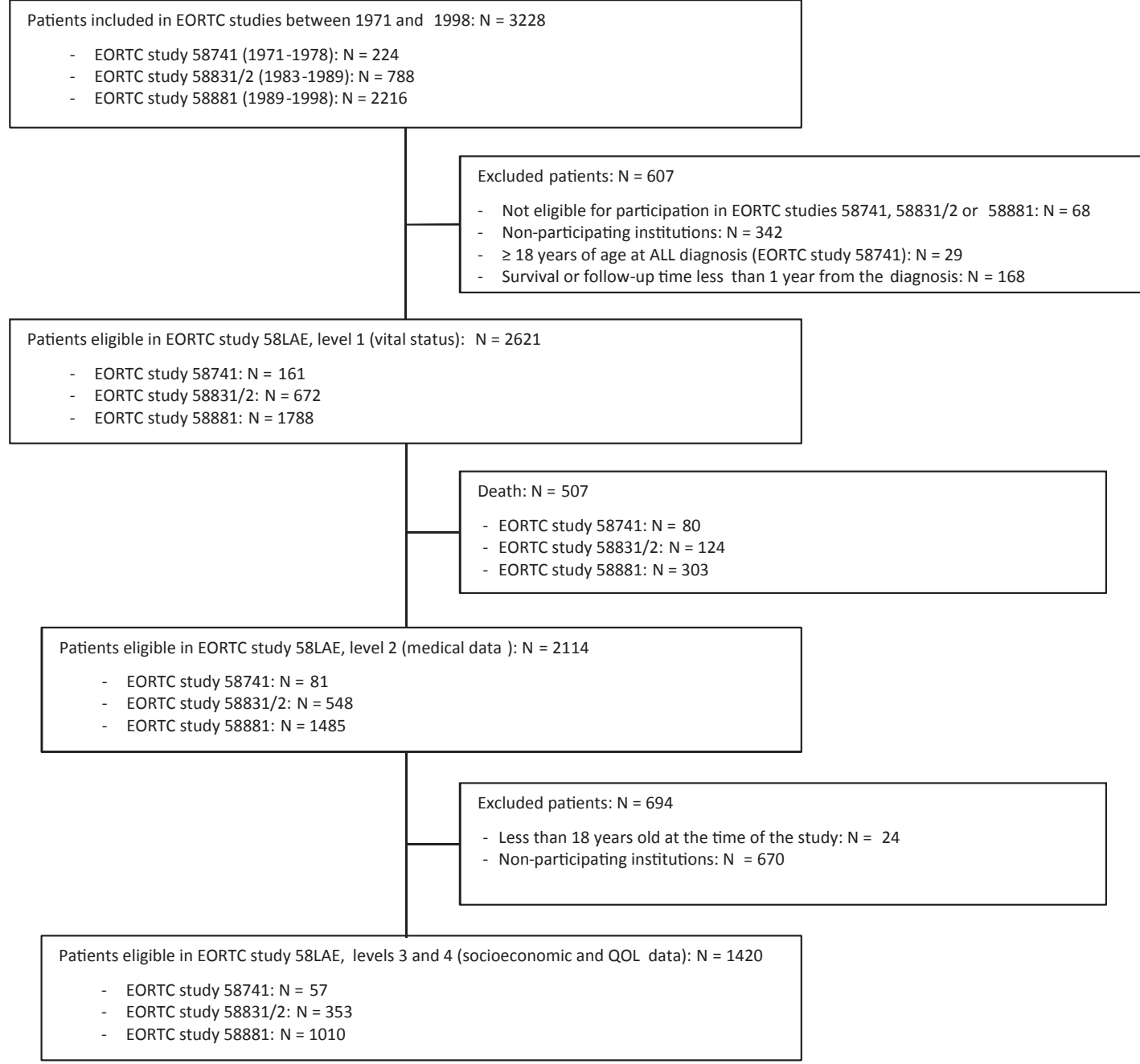

Fig. 1. CONSORT diagram for EORTC study 58LAE.

females had ever had a miscarriage. The incidences of other negative pregnancy outcomes (e.g. birth defect, medical abortion) were low. The average number of children females had was 0.36 (95\% CI: 0.27-0.44). Among males who had tried to have children, $83 \%$ made their partner pregnant, $72 \%$ had children and $7 \%$ used medical help to make their partner pregnant. For both females and males, the incidence of negative pregnancy outcomes other than miscarriage was low.

\section{The challenges}

The global aim of assessing the long-term outcome of a large and multinational cohort of patients is as necessary, as it is arduous. The EORTC study 58LAE is an ambitious, far-reaching project, and as such, it has raised several challenges when translating the initial study design into the "real-life" setting.

Firstly, there is an inherent difficulty in studies that require a prolonged follow-up of patients. In childhood cancer, there is an additional difficulty to track down the steps of the patients, being the transition from children or adolescent and young adults (AYA) units and hospitals to adult oncology facilities a critical point, in which there is a high risk of losing subjects to follow-up. Not only the change of the caregivers' team, but also the natural change of contact details over time, add to the complexity of survivorship studies. Depending on the personal data protection regulation of each country, it is sometimes difficult to access these contact details. Furthermore, the lack of social security numbers in children impairs traceability when they become adults.

Secondly, the inclusion of patients from multiple clinical trials also complicates the statistical analysis of the association between treatment exposures and long-term health effects. A large variety of treatment protocols makes it difficult to distinguish between the effects of specific treatment strategies. Moreover, clinical trials may differ between each

Table 2

Number of available data in the EORTC database for levels 1 (vital status), 2 (medical data), 3 (socioeconomic outcome) and 4 (QOL).

\begin{tabular}{|c|c|c|c|c|c|c|}
\hline \multirow[t]{2}{*}{ 58LAE study levels } & \multirow[t]{2}{*}{ Number of participating institutions } & \multirow{2}{*}{$\begin{array}{l}\text { Number of eligible } \\
\text { patients }\end{array}$} & \multicolumn{4}{|c|}{ Number of available data in the EORTC database (\% eligible patients) } \\
\hline & & & 58741 & $58831 / 2$ & 58881 & Total \\
\hline Level 2: medical data & 32 & 2114 & $77(95.1)$ & $439(80.1)$ & $1160(78.1)$ & $1676(79.3)$ \\
\hline Level 3: socioeconomic outcome & 22 & 1420 & 25 (43.9) & 109 (30.9) & $373(36.9)$ & $507(35.7)$ \\
\hline Level 4: $Q O L$ & 22 & 1420 & $17(29.8)$ & $42(11.9)$ & $134(13.3)$ & $193(13.6)$ \\
\hline
\end{tabular}


other in terms of the period of time when they were performed or other characteristics such as the inclusion criteria of the patients. These differences may introduce confounding that may be difficult to account for. For example, the effect of treatment strategies used more frequently in older trials, like cranial radiotherapy, is confounded by the effect of the age of cancer survivors at the time of the assessment of long-term outcomes.

Thirdly, in order to perform a comprehensive and updated crosssectional assessment of the health and quality of life of a survivors cohort, different approaches or a combination of them can be implemented for data collection. This may vastly increase the funding requirements and adds to the complexity of carrying out long-term outcome studies. Some of these possibilities to gather updated patient information include:

Patient self-reported clinical outcomes, which are easier to access but can lead to incomplete or misleading data;

Medical records, which are certainly more reliable but not always updated;

New clinical and complimentary examinations, which can be too expensive and logistically not feasible for the participating institutions to organize; and

Contacting the general practitioners, which can be problematic from a regulatory and administrative point of view, as explained in the next paragraph.

Fourthly, overcoming the heavy healthcare administrative machinery is certainly a significant challenge. This is more so the case when attempting to gather patient information from the pre-computerization era; old paper medical records are difficult to retrieve from archives. Another aspect of the administrative barriers that need to be dealt with is that data transfer from national registries cannot be centralized by the clinical research organization and must be done instead through the participating institutions. This slows down all study-related procedures, increasing the complexity and the need for resources.

Finally, the patient informed consent of old studies does frequently not foresee new data collection. This shortage is being addressed in the more recent and current studies, and will facilitate future survivorship studies, but it entails additional difficulties for the present ones. However, it is noteworthy that in spite of the high number of patients lost to follow-up, the number of contacted patients refusing to participate is very low, which highlights the weight of administrative and regulatory obstacles over the willingness of participants.

Overall, conducting a long-term outcome study is unquestionably as enriching and rewarding, as it is full of challenges.

\section{The perspectives}

With all the above-explained challenges, new ways of addressing survivorship studies are needed. We have already implemented a novel and multi-angle approach to help collect survivors' data and draw a comprehensive picture of their health and well-being. We have retrieved data from both the healthcare providers and institutions, through medical records and new outcome forms; and from the patients, through socioeconomic and QOL questionnaires.

In addition to this, we are currently evaluating other possibilities for data collection, both for socioeconomic and medical outcomes, through collaborations with national and European cancer registries and administrative databases. Particularly the perspective of cross-matching both sources of information could be a powerful tool to enhance not only the quantity, but also the quality of the data.

Moreover, we hope for a paradigm shift when approaching longterm follow-up of patients. This should no longer be seen as a merely complimentary, even optional aspect of cancer research, but rather as an essential part of clinical trials. This will allow for prospectively planned follow-up strategies, which will certainly improve survivorship studies.

Lastly, worth mentioning are the new possibilities that the digital era has to offer. The use of phone/table apps is already being implemented in the QOL assessment of ongoing clinical trials. It is only a matter of time until these tools become a regular part of daily clinical practice; they might as well be used for long-term follow-up of patients, which would certainly facilitate and improve the quality of survivorship studies.

The importance of non-interrupted follow-up of cancer survivors throughout their life transitions from childhood to adolescence and adulthood is being increasingly recognized by the different healthcare research stakeholders as being of high importance. It is time to address this need and translate it into affordable and well-planned long-term follow-ups in the context of clinical trials.

\section{Acknowledgements}

The authors thank all the EORTC-CLG study group members. We thank the clinicians and biologists who participated in the studies. The authors also thank the EORTC HQ Data Management Department members (Lies Meirlaen, Liv Meert, Gabriel Solbu, Alessandra Busato, Aurélie Dubois, Nicole Duez, Livia Giurgea, Jan Herman, Bénédicte Marchal, Isabel VandeVelde and Christine Waterkeyn) for their essential support. This work was supported by the Kinderkankerfonds (a nonprofit childhood cancer foundation under Belgian law) and by Fonds Cancer (FOCA) from Belgium..

\section{References}

[1] M. Duval, et al., Comparison of Escherichia coli-asparaginase withErwinia-asparaginase in the treatment of childhood lymphoid malignancies: results of a randomized European Organisation for Research and Treatment of Cancer-Children's Leukemia Group phase 3 trial, Blood 99 (2002) 2734-2739.

[2] J. van der Werff Ten Bosch, et al., Value of intravenous 6-mercaptopurine during continuation treatment in childhood acute lymphoblastic leukemia and nonHodgkin's lymphoma: final results of a randomized phase III trial (58881) of the EORTC CLG, Leuk. Off. J. Leuk. Soc. Am. Leuk. Res. Fund, U. K. 19 (2005) 721-726.

[3] B.F. Millot, et al., Value of high-dose cytarabine during interval therapy of a BerlinFrankfurt-Munster - based protocol in increased- risk children with acute lymphoblastic leukemia and lymphoblastic lymphoma: results of the European organization for research and treatment, J. Clin. Oncol. 19 (2010) 1935-1942.

[4] E. Vilmer, et al., Long-term results of three randomized trials (58831, 58832, 58881 ) in childhood acute lymphoblastic leukemia: a CLCG-EORTC report, Leukemia 14 (2000) 2257-2266.

[5] M.A.E. Van Der Kaaij, et al., Parenthood in survivors of Hodgkin lymphoma: an EORTC-GELA general population case-control study, J. Clin. Oncol 30 (2012) 3854-3863.

[6] J.J. Ware, M.M. Kosinski, S.S.D. Keller, A 12-Item Short-Form Health Survey: construction of scales and preliminary tests of reliability and validity, Med. Care 34 (1996) 220-233.

[7] B.J. Zebrack, W. Landier, The perceived impact of cancer on quality of life for posttreatment survivors of childhood cancer, Qual. Life Res 20 (2011) 1595-1608.

[8] G. Dupuis, J. Perrault, M.-C. Lambany, E. Kennedy, P. David, A new tool to assess quality of life: the Quality of Life Systemic Inventory, Qual. Life Cardiovasc. Care 5 (1998) 36-45.

[9] C. Piette, et al., Differential impact of drugs on the outcome of ETV6-RUNX1 positive childhood B-cell precursor acute lymphoblastic leukaemia: results of the EORTC CLG 58881 and 58951 trials, Leukemia 32 (Jan. (1)) (2018) 244-248, http://dx.doi.org/10.1038/leu.2017.289.

[10] C. Piette, et al., Prophylactic CNS therapy (with or without radiation therapy) in medium-high risk acute lymphoblastic leukemia (ALL) children: long-term outcome evaluation of the randomized BFM-oriented trial 58832 (period 1983-1989) of the EORTC children leukemia group, Blood 128 (2016).

[11] M. Barbati, et al., Socioeconomic outcomes among long-Term childhood acute lymphoblastic leukemia survivors enrolled between 1971 and 1998 in the EORTC children leukemia group trials 58741, 58831/2 and 58881, Blood 130 (2017).

[12] T. De Rojas, et al., Fertility status among long-term childhood acute lymphoblastic leukemia survivors enrolled between 1971 and 1998 in the EORTC children leukemia group trials 58741, 58831/2 and 58881, Blood 130 (2017). 